\title{
Findings from the microbiota of tooth apical periodontitis and the search for pathogen forgranulomatous inflammation
}

\section{Yuanyuan Wang}

Fourth Military Medical University School of Stomatology: Air Force Medical University School of Stomatology

\section{Hao Xu}

Xi'an Medical University

\section{Minghui Wei}

Fourth Military Medical University School of Stomatology: Air Force Medical University School of Stomatology

\section{Yuhong Wang}

Fourth Military Medical University School of Stomatology: Air Force Medical University School of

\section{Stomatology}

\section{Wenzhe Wang}

Fourth Military Medical University School of Stomatology: Air Force Medical University School of Stomatology

\section{Bin Feng}

Fourth Military Medical University School of Stomatology: Air Force Medical University School of Stomatology

Jia Ju

Fourth Military Medical University School of Stomatology: Air Force Medical University School of Stomatology

\section{Xinwen Wang ( $\nabla$ xinwen.wang@yahoo.com )}

Fourth Military Medical University School of Stomatology: Air Force Medical University School of Stomatology https://orcid.org/0000-0001-6880-8098

\section{Yuan Liu}

Fourth Military Medical University School of Stomatology: Air Force Medical University School of Stomatology

\section{Research}

Keywords: Apical periodontitis, Granulomatous inflammation, Orofacial granulomatosis, Proteobacteria, Neisseria subflava 
Posted Date: August 10th, 2021

DOl: https://doi.org/10.21203/rs.3.rs-746573/v1

License: (c) (i) This work is licensed under a Creative Commons Attribution 4.0 International License. Read Full License 


\section{Title page:}

Findings from the microbiota of tooth apical periodontitis and the search for pathogen for granulomatous inflammation

Yuanyuan Wang ${ }^{1,2}$, Hao $\mathrm{Xu}^{3}$, Minghui Wei ${ }^{1,2}$, Yuhong Wang ${ }^{1,2}$, Wenzhe Wang ${ }^{1,2}$, Bin Feng ${ }^{2,4}$, Jia $\mathrm{Ju}^{2,4}$, Xinwen Wang ${ }^{1,2}$, Yuan $\mathrm{Liu}^{2,5}$

Authorship note: YYW and HX are co-first authors.

Conflict of interest: The authors declare no potential conflicts of interest with respect to the authorship and publication of this article.

\section{Corresponding author:}

Xinwen Wang or Yuan Liu MD, PhD, Shaanxi Clinical Research Center for Oral Diseases; the National Clinical Research Center for Oral Disease of China; State Key Laboratory of Military Stomatology, School of Stomatology, the Fourth Military Medical University.

Telephone: (086)029-84772604

Fax: (086)029-84772654

Email: xinwen.wang@yahoo.com or liuyuan@fmmu.edu.cn

Address: Changle West Road 145, Xi'an, 710032, Shaanxi Province, China. 
Background: Orofacial granulomatosis (OFG) is a granulomatous inflammation (GI) disease in maxillofacial region, the underlying cause of it remains unknown. Our previous study demonstrated that tooth apical periodontitis (AP) plays a significant role in the pathogenesis of OFG, we aimed here to characterize the AP bacterial signatures of OFG patients, and identify bacteria that may be important pathogens capable of inducing OFG.

Results: The composition of AP microbiota in OFG cases and common AP controls was compared using $16 \mathrm{~S}$ rRNA gene sequencing, the results showed a specific AP microbiota signature in OFG patients, characterized by domination of phyla Firmicutes and Proteobacteria, notably members of Streptococcus, Lactobacillus and Neisseria. To assess the pathogenicity of the potential pathogens in OFG, we isolated and successfully in vitro cultured Streptococcus, Lactobacillus casei, Neisseria subflava, Veillonella parvula and Actinomyces from OFG patients, and injected the clinical isolates into mice respectively. Ultimately, foot pad injection with $N$. subflava elicited granulomatous inflammation, and the virulence of $N$. subflava was verified based on Koch's postulates.

Conclusions: Our findings confirmed the role of bacteria in OFG, and first suggested that the component of the host normal microbiota, $N$. subflava is likely a pathogen for $\mathrm{Gl}$.

Keywords: Apical periodontitis, Granulomatous inflammation, Orofacial granulomatosis, Proteobacteria, Neisseria subflava

\author{
List of abbreviations \\ AP Tooth apical periodontitis \\ CD Crohn's disease \\ GI Granulomatous inflammation \\ LEfSe Linear discriminant analysis effect size \\ LDA Linear discriminant analysis \\ NMDS Non-metric multi-dimensional scaling \\ OFG Orofacial granulomatosis \\ OTU Operational taxonomic unit \\ PCoA Principal coordinate analysis
}




\section{Background}

Orofacial granulomatosis (OFG) is a rare granulomatous inflammation (GI) disease characterized by nontender labial swelling. The disease histologically resembles Crohn's disease (CD), which is a chronic granulomatous inflammatory condition that can affect any part of the gastrointestinal tract[1]. OFG may be idiopathic, localize only in the oral mucosa or in the skin and subcutaneous tissue of the oral cavity, and may also be present in $\operatorname{CD}[2,3]$. A proportion of patients manifesting as OFG at the early stage develop gut $C D$, so the term 'oral $C D$ ' has also been used to describe OFG[4, 5]. More rarely, a small subgroup of OFG patients develop another GI disease, sarcoidosis[6].

Infectious agents have long been considered of playing a role in the initiation of GI disease, so many approaches have been used in the attempt to identify specific microorganisms in the initiation of OFG. Although antibodies against Mycobacterial stress protein[7], Spirochete Borrelia burgdorferi[8] have been found in some patients with OFG, but the results were not confirmed in further study[9]. Until now, a direct causal relationship between microbe and OFG has not yet been established, the underlying cause of OFG remains to be elucidated.

In previous study we found that odontogenic infection has significant effects on the pathogenesis of OFG, 71.5\% OFG patients had chronic tooth apical periodontitis (AP)[2], majority of this kind of OFG patients who received dental treatment showed a marked response, highlighting the involvement of odontogenic infection in the pathophysiology of OFG, microbes in odontogenic infection may be a trigger for the development of OFG. In this study, a comprehensive phylogenetic analysis of AP microbiota from OFG patients was performed, the AP samples from matched individuals without OFG was taken as controls. Thereafter, we isolated and cultured the candidate bacteria, used mouse models to assess the pathogenicity of the candidate bacteria.

\section{Results}

\section{The composition of AP microbiota in OFG patients}

In total, 28 OFG patients with AP (22 female, average age $45.3 \pm 10.2$ years) and 30 age- and gender- matched control individuals ( 20 female, average age $51.7 \pm 16.2$ years) were recruited in the study. The number of final clean reads per sample from OFG patients and control individual ranged from 50416 to 127314 . Those sequences with over $97 \%$ similarity are considered as one operational taxonomic unit (OTU). On average, 4475 OTUs in OFG group and 4899 OTUs in control group have been detected, with 3021 OTUs in common. They were assigned at the phylum, class, order, family and genus taxonomic level. Totally, 6282 OTUs were successfully annotated, which were used for generation of taxonomy tables and rarefaction curves, calculation of species richness and diversity, and clustering of samples with principal coordinate analysis (PCOA).

Figure 1 shows the composition of AP microbiota across all samples. Phyla Bacteroidetes, Firmicutes, Proteobacteria, Fusobacteria and Spirochaetes accounted for the majority of the sequences. In the control group, Bacteroidetes was the most prevalent phylum, followed by Firmicutes, Fusobacteria and Proteobacteria, whereas the OFG group exhibited a specific AP microbiota signiature, Firmicutes was by far the most proportionally abundant phylum, followed by Proteobacteria and Bacteroidetes. Table 1 illustrates the distribution and relative abundance of top 30 genera detected in the two groups. Genera Streptococcus was highly abundant (12-fold 
increase) in the OFG group when compared to that in the control group, Lactobacillus, Neisseria, Gemella, Stenotrophomonas, Salinimicrobium, Granulicatella, Actinobacillus and Aggregatibacter also showed similar overabundance in OFG group. In contrast, Genera Porphyromonas, Fusobacterium, unidentified Spirochaetaceae, and Prevotella spp. accounted for the majority of the bacterial community in the control group.

We also analyzed the microbial community in OFG biopsy tissue. Due to poor DNA quality, three samples from paraffin-embedded biopsy were excluded. Ultimately, 2483 OTUs in six OFG biopsy samples were detected. Figure S1 shows the composition of the microbial communities among the six biopsy samples, Proteobacteria (14.9\%-58.4\%) was the most abundant phylum, followed by Firmicutes (19.5-37.5\%) and Bacteroidetes (4.5\%-21.0\%). At the genus level, the communities in six samples are shown in Table S1. Lactobacillus, Streptococcus and Pseudomonas were prevalent across all the samples. It was noting that in patient 6 sample, the relative abundance of Neisseria was much higher (34.34\%) than any other genus in all samples.

\section{The diversity of AP microbiota in OFG patients}

Bacterial richness and diversity were assessed by Shannon, Chao 1 and Simpson index. The data showed a significantly decreased richness and a -diversity in the OFG group compared with the control $(P<0.01)$ (Fig 2). The results of PCoA analysis based on weighted UniFrac distance matrix and unweighted UniFrac distance matrix, non-metric multi-dimensional scaling (NMDS) analysis, and Anosim statistical analysis all showed that the AP bacterial community in the OFG group was significantly different from that in the control group, they tended to form separated clusters and could be distinguished from each other. The OFG samples appeared more dispersive than control samples (Fig 2).

The differentially abundant class, order, family and genus between the two groups are displayed in Figure 3. T-test results showed that Phyla Firmicute and Proteobacteria were significantly overrepresented in the OFG group $(P<0.001$ and $P=0.008$ respectively) compared with that in the control group ( $\mathrm{Fig} 3 \mathrm{~A}$ ). The cladogram demonstrates the taxa with different abundances among the two groups (Fig 3B). Linear discriminant analysis effect size (LEfSe) was used to predict biomarker taxa in each group, results showed that at the Class level, Bacilli and Gammaproteobacteria were significantly more enriched in the OFG group. In contrast, Bacteroidia, Fusobacterila, Spirochaetia, Clostridia and Synergistia were significantly overabundant in the control group. At the genus level, the OFG group was enriched for Streptococcus and Gemella, whereas the control group was enriched for Porphyromonas, Fusobacterium, Spirochaetaceae, Tannerella and unidentified Prevotellaceae (Fig 3C).

\section{AP microbiota in OFG patients and control individuals exhibit different enrichment in metabolic pathways}

In the present study, there were considerable AP microbiota associated KEGG gene function changes in OFG patients. At KEGG level 2, we identified 31 different KEGG pathways that were significantly different between the OFG group and control group (Fig 4). The top 6 pathways significantly enriched in the OFG group were membrane transport $(P<0.001)$, signal transduction $(P<0.001)$, Xenobiotics biodegradation/metabolism $(P<0.001)$, carbohydrate metabolism $(P=0.006)$, amino acid metabolism $(P=0.045)$ and lipid metabolism $(P=0.001)$. The top 6 pathways significantly enriched in the control group were translation $(P<0.001)$, replication/repair $(P=0.005)$, metabolism of cofactors/vitamins $(P<0.001)$, nucleotide metabolism $(P<0.001)$, 
transport/catabolism $(P<0.001)$ and energy metabolism $(P=0.003)$.

\section{Acquisition of challenge candidate bacteria from OFG patients}

As showed in figure 5, bacterial samples were obtained from the tooth root surface of three OFG patients respectively (age 45 female, age 53 female, age 53 male), and were initially cultured. Thirty isolates with unique color or morphology, in addition to many colonies with similar color or morphology incubated under different conditions were identified to genus by direct sequencing of the 165 gene.

Out of the 30 isolates examined, Streptococcus had the highest percentage occurrence of $100 \%$, followed by Neisseria with $85.7 \%$, Actinomyces $71.4 \%$, Haemophilus $71.4 \%$, Veillonella $71.4 \%$, Romboutsia 64.3\%, Blautia 57.1\%, Escherichia 57.1\%, Faecalibacterium 57.1\% and Lactobacillus 42.9\% percentage occurrence (Table S2). Individual colonies were subcultured to ensure purity. After sequences identification and compare to known sequences using a Blast search of GenBank, ultimately, pure cultures of Streptococcus, Neisseria subflava, Lactobacillus casei, Veillonella parvula, Actinomyces were established (Fig 5).

\section{Effects of candidate bacteria infections in mice}

Clinical isolates of Streptococcus, N. subflava, L. casei, V. parvula, Actinomyces were used for experimental infection of mice. Wild-type $(\mathrm{Wt}) \mathrm{BALB} / \mathrm{c}$ mice were injected with various organisms in hind legs and foot pads respectively. After 20 days, the mice injected with Streptococcus, $L$. casei, $V$. parvula, Actinomyces showed a relatively normal histomorphology, although a few demonstrated sparse inflammatory cell infiltration. The mice infected with $N$. subflava in foot pads yielded histopathology similar to that seen in human OFG. Isolates were recovered from the mice, and the GPCR targeting the gene encoding aspartate semialdehyde dehydrgenase[10] revealed that positive samples contained 4 to 7 times as many N. subflava as other groups (Fig S2).

\section{Discussion}

The mouth is the gateway of the body to the external world and represents one of the most biologically complex sites in the body. The oral microbiota composition is readily changeable by diet, site, temperature, different oral disease condition and other life events. The complexity of the oral microbiota is a significant challenge for analysis and interpretation of the alteration in oral bacterial community, which also partly underlines previous inconsistent results regarding salivary microbiota associated with OFG[11, 12]. AP is a common inflammatory disorder of the dental periradicular tissue, which results from infections of the root canal of endodontically involved teeth[13]. In this study, we collected the microbial samples from the root surface of extracted tooth with AP, avoiding contamination from the oral cavity and reducing the effect of influence factors (including oral hygiene, diet, site, temperature, general oral health status) as low as possible. The results of the current study, performed on 30 teeth with common AP from the individuals without OFG are largely consisted with that found in previous studies[14-16]. It showed that the root bacterial community in AP is relatively stable, which ensured the reliability of the analysis. Together with the fact that AP is a high risk factor for OFG[2], AP microbiota provided an ideal target for the research in this study.

According to previous study, compared with the salivary microbiota in healthy status, high bacterial richness is usually associated with poor oral health[17, 18]. Our results indicated that 
the richness and diversity of AP bacterial communities in OFG patients was significantly decreased, which may attribute to the control we chose. In this study, we took the tooth root microbiota in patients diagnosed as AP but without OFG as control, which may make the diversity of AP bacterial community in OFG appearing to be relatively lessened. However, this decreased diversity consisted with the observation in CD. Many studies have reported that the most consistent change of gut microbiota in CD is a reduction in the diversity[19-21]. Moreover, a relative increase in Proteobacteria was observed in CD patients, in particular on mucosa associated microbiota[22, 23], which is in line with our results.

Our study demonstrated that tooth roots with AP in OFG patient harbor a microbiota that differ significantly from that in study control, phyla Proteobacteria and Firmicutes were dominated instead of phyla Bacteroidetes and Fusobacteria, which are usually most abundant in common AP microbiota[15, 16]. This result was further confirmed in OFG biopsy sections. It is noteworthy, Gammaproteobacteria was shown significantly correlated with OFG (Fig 3). As a member of Gammaproteobacteria (https://www.arb-silva.de/), Neisseria was found to be one of the top enriched taxa in both AP microbiota and biopsy tissue of OFG patients, highlighting its possible role in OFG pathogenesis. We isolated $N$. subflava from the root surface with AP in OFG patients, and injected the clinical isolate into animal model. GI responses were observed in mice foot pad, moreover $N$. subflava could be detected, and were obviously abundant in pathologic lesions in these mice, which fulfilled Koch's postulates and strongly suggested the pathogenicity of $N$. subflava in OFG.

Proteobacteria are one of the most abundant phyla within human body, which are divided into six classes based on phylogenetic analysis of 16S rRNA, (alpha-, beta-, gamma-, delta-, epsilonand zeta-Proteobacteria)[24]. There are many Gammaproteobacteria members, including Escherichia coli[25], Francisella tularensis[26], Yersinia[27], Salmonella typhi[28] and Burkholderia[29] have been reported to be provocative factors for the development of a range of GI diseases. Genus Neisseria belonging to Gammaproteobacteria are comprised of Gram-negative species, which are thought to be restricted to human generally[30]. Most of Neisseria spp. are regarded as components of the host normal microbiota[31], and might occasionally behave as opportunistic pathogens[32]. However, there is increasing evidence to suggest that these 'harmless inhabitants' are capable of producing infection in a wide variety of anatomical sites[31], e.g. granulomatous inflammation in the patients with immunodeficiency[33, 34]. Whether certain vertical inheritance shared among Gammaproteobacteria play a role in the occurrence of $\mathrm{Gl}$ diseases is an interesting question to be answered in the future.

Clinically, the course of AP is usually extremely painful or hypersensitivity. Yet in our previous study, we noticed that the AP in OFG patients is asymptomatic without experience of sharp and severe pain[2]. Previous microbiological and molecular studies showed that fermentation end products of various bacteria in deep caries are close related with clinical pain or hypersensitivity[35], the carious teeth with high lactobacillus counts are not sensitive to stimuli $[36,37]$, as the organic acids produced by lactobacillus not only fail to excite the intratubular A- $\delta$ nerves but also suppress nerve impulses elicited by other stimuli[38]. In the present study, we found the Lactobacillus was consistently enriched in AP samples and biopsy tissues of OFG patients. This finding may explain why the AP in OFG patients is asymptomatic, even several teeth involved at the same time. The injection of Lactobacillus did not induce GI response in mice, suggesting a possibility that it might play a synergy role in the pathogenesis of 
OFG.

Increase in abundance of Streptococcus in the salivary microbiota of OFG patients has been reported[12], but reductions have also been reported[11], so the role of Streptococcus in OFG pathogenesis is obscure. In our study, Firmicutes was the most abundant phylum in OFG group, and Streptococcus spp. made the major contribution to the increased proportions, but we did not observe GI reaction in animal model. Liu et al searched for likely bacterial agents in CD tissues using immunocytochemical techniques and found that $44 \%$ of the cases contained the Streptococcal antigen[39]. As Streptococcal immunoreactivity was also found in some normal intestine, so he concluded that Streptococci more than likely act as secondary rather than primary agents. But in Sartor's[40] research, injection of Streptococcal cell wall fragments into the small intestine and cecum was shown to induce granulomatous enterocolitis in rats, reminding us its role should be paid more attention.

In this study, only six potential bacteria species were isolated and evaluated in the animal model, we cannot exclude the possibility that other distinct bacteria contribute to the initiation of OFG. More recently, microbial dysbiosis in commensal community has being given a greater emphasis, different bacteria may take on different roles in disease progression. In this study, the functional analysis revealed enriched pathways related to membrane transport, signal transduction, Xenobiotics biodegradation/metabolism, carbohydrate metabolism, amino acid metabolism and lipid metabolism in OFG. Combining the metabolites and microbiota analyses can help to address questions related to global changes in the microbial community during OFG development.

\section{Conclusions}

OFG patients exhibit a specific AP microbiota signature, characterized by domination of Firmicutes and Proteobacteria. Of them, Neisseria consistently enriched in AP microbiota and OFG lesion tissue, more importantly, the clinical isolate of $N$. subflava from the OFG patients can lead to GI in mice, suggesting its pathogenicity in OFG. Moreover, elevated abundance of lactobacillus may be the main reason for asymptomatic AP in OFG patients and might play a synergy role in the pathogenesis of OFG. Despite high abundance of Streptococcus being found in OFG group, we did not observe GI reaction in animal model, its role need to be elucidated further. This study shade light on the role of bacteria in OFG initiation, opening a novel insight into GI disease research.

\section{Methods}

\section{Study participants}

Eligible case subjects were identified from the National Clinical Research Center for Oral Disease of China. The study was approved by the Ethics committee of the School of Stomatology, the Fourth Military Medical University (approval \# IRB-REV2020039). All participants were newly diagnosed and signed an informed consent form prior to their enrolment in the study. The OFG patients fulfilled the diagnostic criteria for OFG according to currently accepted critieria[2]. The patients diagnosed as AP but without OFG at the same period were recruited as control. Complete medical history and panoramic radiograms of each patient were assessed. All participants were selected by applying the following exclusion criteria: hematologic diseases, uncontrolled diabetes, or disease of the immune system, and were treated by topical/systemic 
antibiotics or immune agents within one month. Teeth were selected if they were clinically diagnosed as AP and required extraction because they were judged unrestorable.

\section{DNA extraction and 165 rRNA sequencing}

Prior to tooth extraction, all participants were given sterile physiological saline solution to rinse for one minute. Immediately after extraction, microbial samples were taken from the apical part of root with sterile swab, always trying to avoid saliva contamination, and frozen at $-20^{\circ} \mathrm{C}$.

DNA was extracted using the CTAB/SDS method. The V3-4 region of the bacterial 16S rRNA genes was amplified used specific primer (338F-806R). For the formalin-fixed, paraffin-embedded tissue, five slices of tissue samples were used for total genomic DNA extraction, specific primer (515F-806R) was used for the amplification of the V4 region of bacterial 16S rRNA gene. After 16S rDNA library preparation and generation, the library quality was assessed on the Qubit@2.0 Fluorometer (Thermo Scientific) and Agilent Bioanalyzer 2100 system. The library was sequenced on an Illuminal HiSeq2500 platform, and 250-bp paired-end reads were generated.

\section{Taxonomic analysis}

Sequences analysis was performed using Uparse software (Uparse $v$ 7.0.1001). Sequences with $>97 \%$ similarity were assigned to the same OTUs, the Silva Database was used based on the RDP classifier algorithm to annotate taxonomic information. OTUs abundance information was normalized using a standard of sequence number corresponding to the sample with the lease sequences.

Alpha and beta diversity basing on this output normalized data were used to compare the differences of AP microbiota in OFG patients and control individuals. Unweighted UniFrac measures the distance between two bacterial communities by calculating the fraction of the branch length in a phylogenetic tree. Weighted UniFrac measures the distance between two bacterial communities by comparing the genetic diversity within each community to the total genetic diversity of the communities combined.

\section{Statistical analysis}

Data analysis was performed with QIME workflow. The Wilcoxon, $t$-test were used to evaluate the differences in the bacterial populations between the OFG group and control group. For analysis of diversity within samples, diversity indices Chao 1 , Shannon and Simpson were determined. To assess differences of bacterial community between the two groups, principal component analysis (PCA) based on the OTUs, PCoA based on UniFrac and NMDS were performed. Anosim test was run on the distance matrix to obtain statistical confidence for the sample grouping observed by PCOA. LEfSe software was used to analyse differentially abundant taxa, the filter value of the LDA score was 4 by default, $P$ value $<0.05$.

\section{Functional predictions}

Tax4Fun software package was used to predict the functional capabilities of bacterial communities on the base of SILVA-labeled OUT abundances. The SILVA-based 16S rRNA datasets was transformed to a taxonomic profile of the prokaryotic KEGG organisms, which were then normalized by the $16 \mathrm{~S}$ rRNA copy number. The normalized taxonomic abundances were used to predict the functional profile of the bacterial community.

\section{Bacterial isolation and culture}

The apical content was harvested from the extracted teeth of OFG patients using sterile swab, 
and was placed in sterile tube avoiding possible microorganism contamination. The bacterial culture of the inflammatory content was performed on various media plates under different conditions. Plates of brain heart infusion (BHI) agar and $\mathrm{BHI}$ agar with $5 \%$ sheep blood were incubated at $35^{\circ} \mathrm{C}$ under anaerobic $\left(80 \% \mathrm{~N}_{2}, 10 \% \mathrm{H}_{2}, 10 \% \mathrm{CO}_{2}\right)$ and microaerobic condition $(5 \%$ $\mathrm{O}_{2}, 12 \% \mathrm{CO}_{2}$ ) at the same time. Single colonies were subcultured and further enriched on agar plates.

Presumptive identification of specific bacterial isolates that was different in appearance was accomplished by direct sequencing of the $16 \mathrm{~S}$ gene. The 16S rRNA gene of each kind of colony was sequenced using the MicroSeq Full Gene 16S rRNA Bacterial Isolation Sequencing Kit (Applied Biosystems). The complete genomes of isolates were determined and were used to identify isolates to species.

\section{Animal experiments}

Wild-type (Wt) BALB/c were bred and maintained in a specific pathogen-free environment at the Animal Care Center of the School of Stomatology, FMMU. All experiments were approved by the Institute's Animal Care and Use Committee (No. 20200903). The 4-wk-old male mice weighted from 20 to $30 \mathrm{~g}$ were used for experiments.

The pure cultures of bacteria identified through 16S rRNA sequencing served as the source of bacteria used in the animal study. After bacteria were harvested by centrifugation, the bacterial pellet was diluted to $1 \times 10^{8}$ cells $/ \mathrm{ml}$. For a bacteria species, three mice were injected with $0.5 \mathrm{ml}$ bacteria suspension into the foot pads, three mice were injected same aliquot into the hind legs. At 20 days after injection, mice were sacrificed. Pads and legs were cut sagittal, samples were obtained for bacterial isolation, tissues were fixed with $10 \%$ neutral-buffered formalin and embedded in paraffin. Sections of $5 \mu \mathrm{m}$ thick were prepared and mounted on glass slides. The sections were stained with $\mathrm{H} \& \mathrm{E}$, and subjected to histopathological examination. For the GI positive samples, DNA was extracted from the tissue, the amount of candidate bacteria was determined by probe-based quantitative real-time PCR (q-PCR).

\section{Declarations}

Ethics Approval and Consent to participate

The study protocol was approved by the Ethics Committee of the School of Stomatology, the Fourth Military Medical University (approval \# IRB-REV2020039). Animal experimentation was approved by the Institute's Animal Care and Use Committee (No. 20200903) of the Fourth Military Medical University.

Consent for publication

Not applicable

Availability of data and materials

All data generated or analyzed during this study are included in this article and its supplementary information files. Sequences are deposited at Novo database under the accession X101SC19051730.

\section{Competing interests}

The authors declare that they have no competing interests.

\section{Funding}

This work was supported by grants from the State Key Laboratory of Military Stomatology (2018ZB01) and the National Clinical Research Center for Oral Disease of China (LCA202008). 


\section{Authors' contributions}

WYY and $\mathrm{XH}$ performed the experiments, analyzed the data and draft the manuscript. WYH, WMH and WWZ performed the initial experiments and analyzed the data. FB and JJ assisted with all the experiments, critically revised the manuscript. WXW and LY contributed to conception, design, interpretation of the study, and supervised project planning. All authors visualized data, reviewed and edited the manuscript.

\section{Acknowledgments}

We would like to thank all of the study participants and Professor Haotai Chen and Professor Yinlan Bai for their precious suggestions on microbiota analyses and their comments on the work.

\section{Authors' information}

${ }^{1}$ Department of Oral Medicine, School of Stomatology, the Fourth Military Medical University (FMMU), Xi'an, China; ${ }^{2}$ Shaanxi Clinical Research Center for Oral Diseases, the National Clinical Research Center for Oral Disease of China, State Key Laboratory of Military Stomatology; ${ }^{3}$ Shaanxi Key Laboratory of Brain Disorders \& School of Basic Medical Sciences, Xi'an Medical University, Xi'an, China; ${ }^{4}$ Department of Pharmacy, School of Stomatology, FMMU; ${ }^{5}$ Department of Oral Histology and Pathology, School of Stomatology, FMMU. 


\section{References}

1. Gajendran M, Loganathan P, Catinella AP, Hashash JG: A comprehensive review and update on Crohn's disease. Dis Mon 2018, 64(2):20-57.

2. Wei MH XC, Liu YB, Wang YH, Wang YY, Wang XW, Liu Y.: Characterizing disease manifestations and treatment outcomes among patients with orofacial granulomatosis in China. JAAD International 2020, 1(2):126.

3. Camacho F, Garcia-Bravo B, Carrizosa A: Treatment of Miescher's cheilitis granulomatosa in Melkersson-Rosenthal syndrome. J Eur Acad Dermatol Venereol 2001, 15(6):546-549.

4. Campbell H, Escudier M, Patel P, Nunes C, Elliott TR, Barnard K, Shirlaw P, Poate T, Cook R, Milligan $\mathrm{P}$ et al: Distinguishing orofacial granulomatosis from crohn's disease: two separate disease entities? Inflamm Bowel Dis 2011, 17(10):2109-2115.

5. Sanderson J, Nunes C, Escudier M, Barnard K, Shirlaw P, Odell E, Chinyama C, Challacombe S: Oro-facial granulomatosis: Crohn's disease or a new inflammatory bowel disease? Inflamm Bowel Dis 2005, 11(9):840-846.

6. Al-Hamad A, Porter S, Fedele S: Orofacial Granulomatosis. Dermatol Clin 2015, 33(3):433-446.

7. Ivanyi L, Kirby A, Zakrzewska JM: Antibodies to mycobacterial stress protein in patients with orofacial granulomatosis. J Oral Pathol Med 1993, 22(7):320-322.

8. Liu H, Zheng L: Spirochetes--the possible etiological factor of the cheilitis granulomatosa. Chin Med Sci J 2001, 16(1):52-55.

9. Muellegger RR, Weger W, Zoechling N, Kaddu S, Soyer HP, El Shabrawi-Caelen L, Kerl H: Granulomatous cheilitis and Borrelia burgdorferi: polymerase chain reaction and serologic studies in a retrospective case series of 12 patients. Arch Dermatol 2000, 136(12):1502-1506.

10. Jung JY, Yoon HK, An S, Lee JW, Ahn ER, Kim YJ, Park HC, Lee K, Hwang JH, Lim SK: Rapid oral bacteria detection based on real-time PCR for the forensic identification of saliva. Sci Rep 2018, 8(1):10852.

11. Liu Y, Zhang $\mathrm{Q}, \mathrm{Hu} \mathrm{X}$, Chen $\mathrm{F}, \mathrm{Hua} \mathrm{H}$ : Characteristics of the salivary microbiota in cheilitis granulomatosa. Med Oral Patol Oral Cir Bucal 2019, 24(6):e719-e725.

12. Goel RM, Prosdocimi EM, Amar A, Omar Y, Escudier MP, Sanderson JD, Wade WG, Prescott NJ: Streptococcus Salivarius: A Potential Salivary Biomarker for Orofacial Granulomatosis and Crohn's Disease? Inflamm Bowel Dis 2019, 25(8):1367-1374.

13. Kakehashi S, Stanley HR, Fitzgerald RJ: The Effects of Surgical Exposures of Dental Pulps in Germ-Free and Conventional Laboratory Rats. Oral Surg Oral Med Oral Pathol 1965, 20:340-349.

14. Sundqvist G: Associations between microbial species in dental root canal infections. Oral Microbiol Immunol 1992, 7(5):257-262.

15. Sassone LM, Fidel R, Faveri M, Fidel S, Figueiredo L, Feres M: Microbiological evaluation of primary endodontic infections in teeth with and without sinus tract. Int Endod J 2008, 41(6):508-515.

16. Bouillaguet S, Manoil D, Girard M, Louis J, Gaia N, Leo S, Schrenzel J, Lazarevic V: Root Microbiota in Primary and Secondary Apical Periodontitis. Front Microbiol 2018, 9:2374.

17. Yamashita Y, Takeshita T: The oral microbiome and human health. J Oral Sci 2017, 
59(2):201-206.

18. Takeshita T, Kageyama S, Furuta M, Tsuboi H, Takeuchi K, Shibata Y, Shimazaki Y, Akifusa S, Ninomiya T, Kiyohara $Y$ et al: Bacterial diversity in saliva and oral health-related conditions: the Hisayama Study. Sci Rep 2016, 6:22164.

19. Sheehan D, Moran C, Shanahan F: The microbiota in inflammatory bowel disease. J Gastroenterol 2015, 50(5):495-507.

20. Walker AW, Sanderson JD, Churcher C, Parkes GC, Hudspith BN, Rayment N, Brostoff J, Parkhill J, Dougan G, Petrovska L: High-throughput clone library analysis of the mucosa-associated microbiota reveals dysbiosis and differences between inflamed and non-inflamed regions of the intestine in inflammatory bowel disease. BMC Microbiol 2011, 11:7.

21. Manichanh C, Rigottier-Gois L, Bonnaud E, Gloux K, Pelletier E, Frangeul L, Nalin R, Jarrin C, Chardon P, Marteau $\mathrm{P}$ et al: Reduced diversity of faecal microbiota in Crohn's disease revealed by a metagenomic approach. Gut 2006, 55(2):205-211.

22. Martinez C, Antolin M, Santos J, Torrejon A, Casellas F, Borruel N, Guarner F, Malagelada JR: Unstable composition of the fecal microbiota in ulcerative colitis during clinical remission. Am J Gastroenterol 2008, 103(3):643-648.

23. Swidsinski A, Ladhoff A, Pernthaler A, Swidsinski S, Loening-Baucke V, Ortner M, Weber J, Hoffmann $U$, Schreiber S, Dietel $M$ et al: Mucosal flora in inflammatory bowel disease. Gastroenterology 2002, 122(1):44-54.

24. Rizzatti G, Lopetuso LR, Gibiino G, Binda C, Gasbarrini A: Proteobacteria: A Common Factor in Human Diseases. Biomed Res Int 2017, 2017:9351507.

25. Barnich N, Darfeuille-Michaud A: Adherent-invasive Escherichia coli and Crohn's disease. Curr Opin Gastroenterol 2007, 23(1):16-20.

26. Lamps LW, Havens JM, Sjostedt A, Page DL, Scott MA: Histologic and molecular diagnosis of tularemia: a potential bioterrorism agent endemic to North America. Mod Pathol 2004, 17(5):489-495.

27. Pal K: Granulomatous appendicitis in children: a single institutional experience. Afr $J$ Paediatr Surg 2014, 11(1):26-31.

28. Muniraj K, Padhi S, Phansalkar M, Sivakumar P, Varghese RG, Kanungo R: Bone marrow granuloma in typhoid Fever: a morphological approach and literature review. Case Rep Infect Dis 2015, 2015:628028.

29. Langer S, Radhakrishnan N, Pradhan S, Das J, Saraf A, Kotwal J: Clinical and Laboratory Profiles of 17 Cases of Chronic Granulomatous Disease in North India. Indian J Hematol Blood Transfus 2021, 37(1):45-51.

30. Liu G, Tang CM, Exley RM: Non-pathogenic Neisseria: members of an abundant, multi-habitat, diverse genus. Microbiology (Reading) 2015, 161(7):1297-1312.

31. Humbert MV, Christodoulides M: Atypical, Yet Not Infrequent, Infections with Neisseria Species. Pathogens 2019, 9(1).

32. Herbert DA, Ruskin J: Are the "nonpathogenic" Neisseriae pathogenic? Am J Clin Pathol 1981, 75(5):739-743.

33. Claassen JL, Eppes SC, Buckley RH: Pulmonary coin lesion caused by Neisseria mucosa in a child with chronic granulomatous disease. Pediatr Infect Dis J 1987, 6(6):567-569.

34. Washburn RG, Bryan CS, DiSalvo AF, Macher AM, Gallin JI: Visceral botryomycosis caused by Neisseria mucosa in a patient with chronic granulomatous disease. J Infect Dis 1985, 
151(3):563-564.

35. Hahn CL, Liewehr FR: Relationships between caries bacteria, host responses, and clinical signs and symptoms of pulpitis. J Endod 2007, 33(3):213-219.

36. Hahn CL, Falkler WA, Jr., Minah GE: Correlation between thermal sensitivity and microorganisms isolated from deep carious dentin. J Endod 1993, 19(1):26-30.

37. Hahn CL, Falkler WA, Jr., Minah GE: Microbiological studies of carious dentine from human teeth with irreversible pulpitis. Arch Oral Biol 1991, 36(2):147-153.

38. Panopoulos $\mathrm{P}$, Mejare B, Edwall L: Effects of ammonia and organic acids on the intradental sensory nerve activity. Acta Odontol Scand 1983, 41(4):209-215.

39. Liu Y, van Kruiningen HJ, West AB, Cartun RW, Cortot A, Colombel JF: Immunocytochemical evidence of Listeria, Escherichia coli, and Streptococcus antigens in Crohn's disease. Gastroenterology 1995, 108(5):1396-1404.

40. Sartor RB, Cromartie WJ, Powell DW, Schwab JH: Granulomatous enterocolitis induced in rats by purified bacterial cell wall fragments. Gastroenterology 1985, 89(3):587-595. 


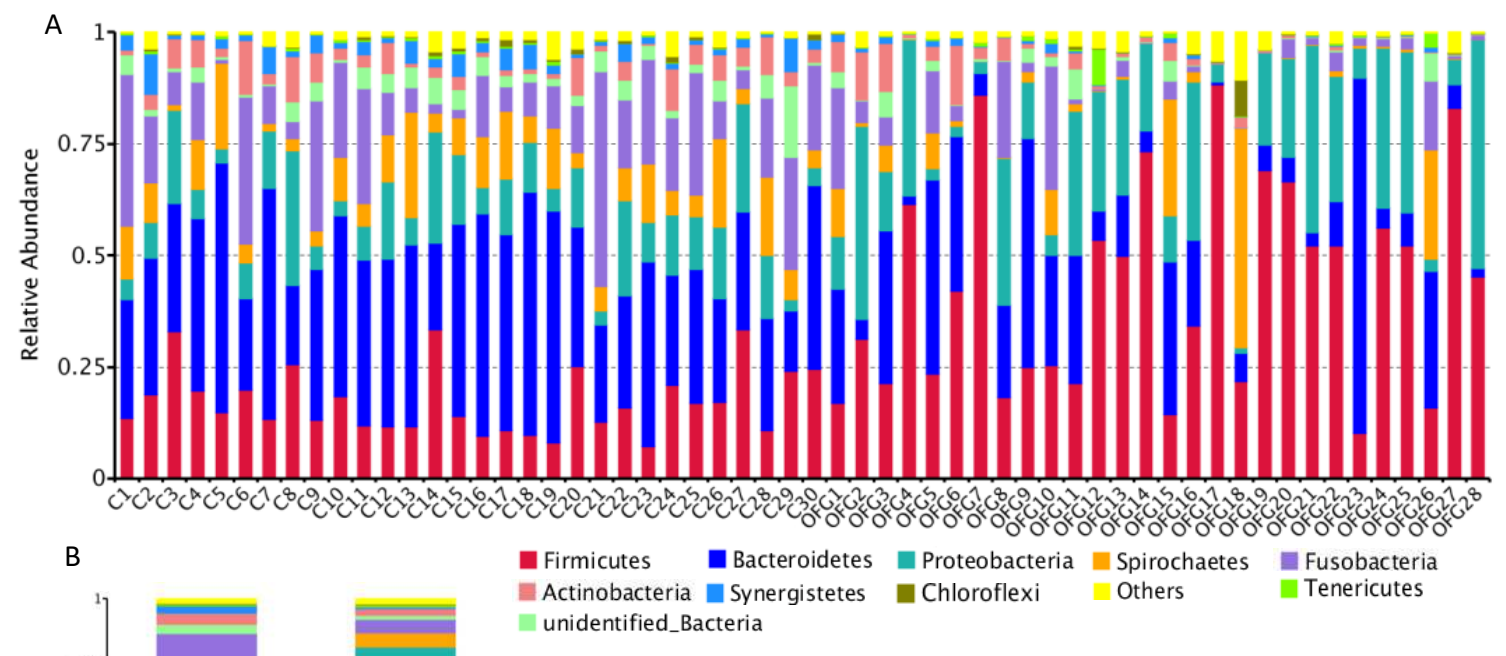

Figure 1. The composition of AP microbiota in OFG patients and control individuals. (A) Relative abundances of the top 10 phyla identified in all OFG patients and control individuals. (B) Relative abundance of the top 10 phyla in the different groups. The height represents the percentage of phylum. AP, apical periodontitis; OFG, orofacial granulomatosis. 

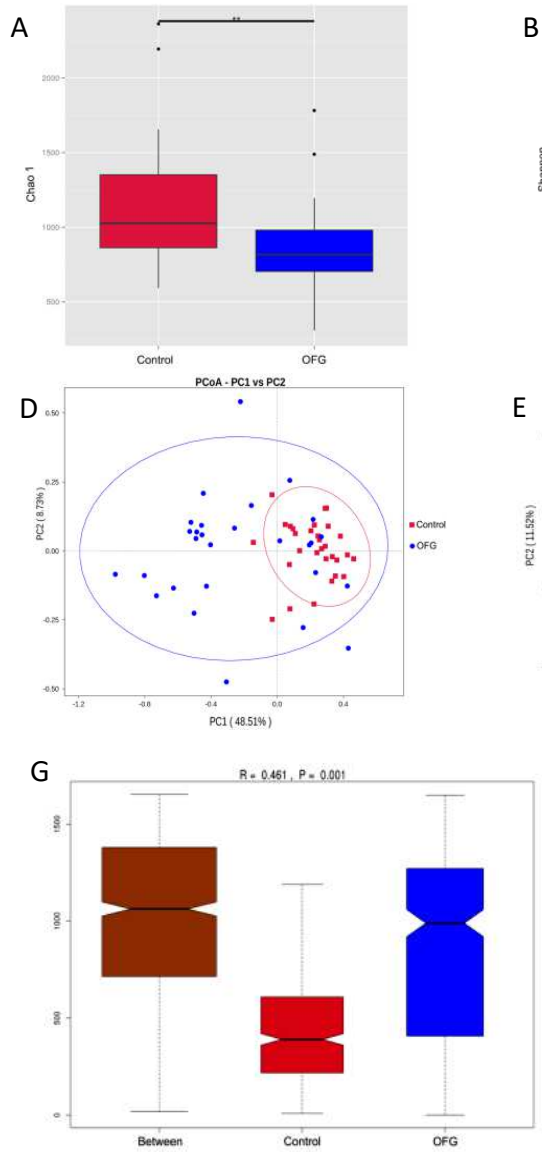

B
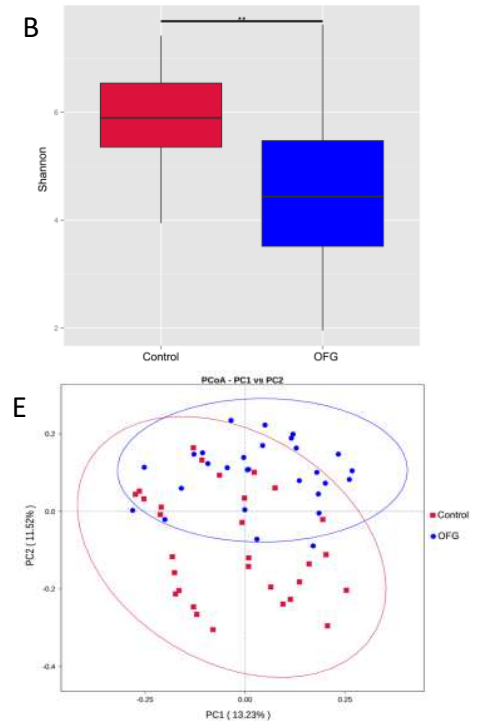

C

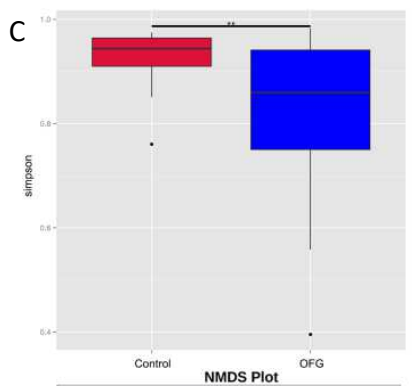

F

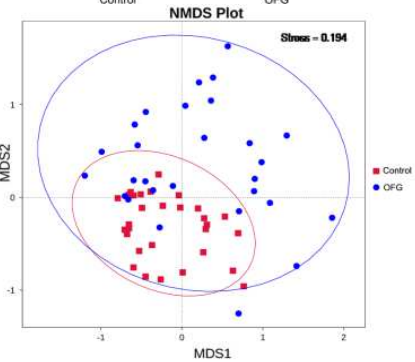

Figure 2. Alpha diversity and Bata diversity analysis of AP microbiota in OFG patients and control individuals. (A) Chao 1 (B) Shannon (C) Simpson index was calculated based on OUT relative abundance. (D) Clusters formed by PCOA based on weighted UniFrac distance matrix. Each dot represents a sample. (E) Clusters formed by PCoA based on unweighted UniFrac distance matrix. (F) Clusters formed by NMDS analysis. (G) Anosim statistical analysis showed a significant difference between the OFG and control group ( $R=0.461, P=0.001)$. ${ }^{* *} 0.001<P<0.01$. AP, apical periodontitis; OFG, orofacial granulomatosis; PCoA, principle coordinate analysis; NMDS, nonmetric multi-dimensional scaling. 
A

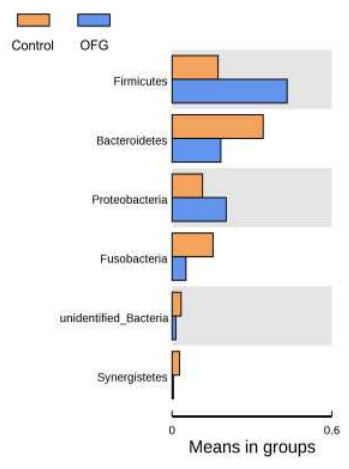

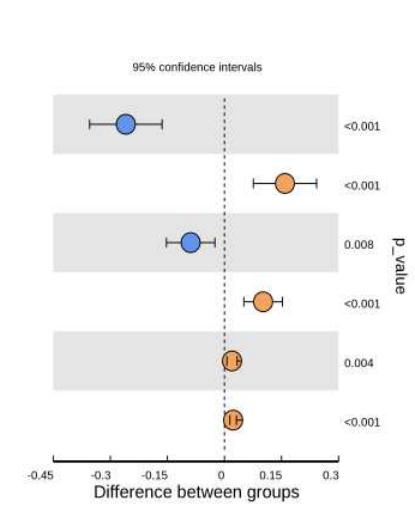

B

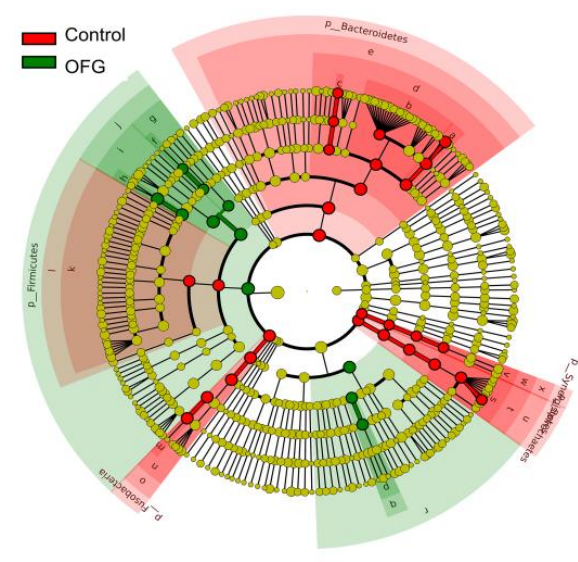

C

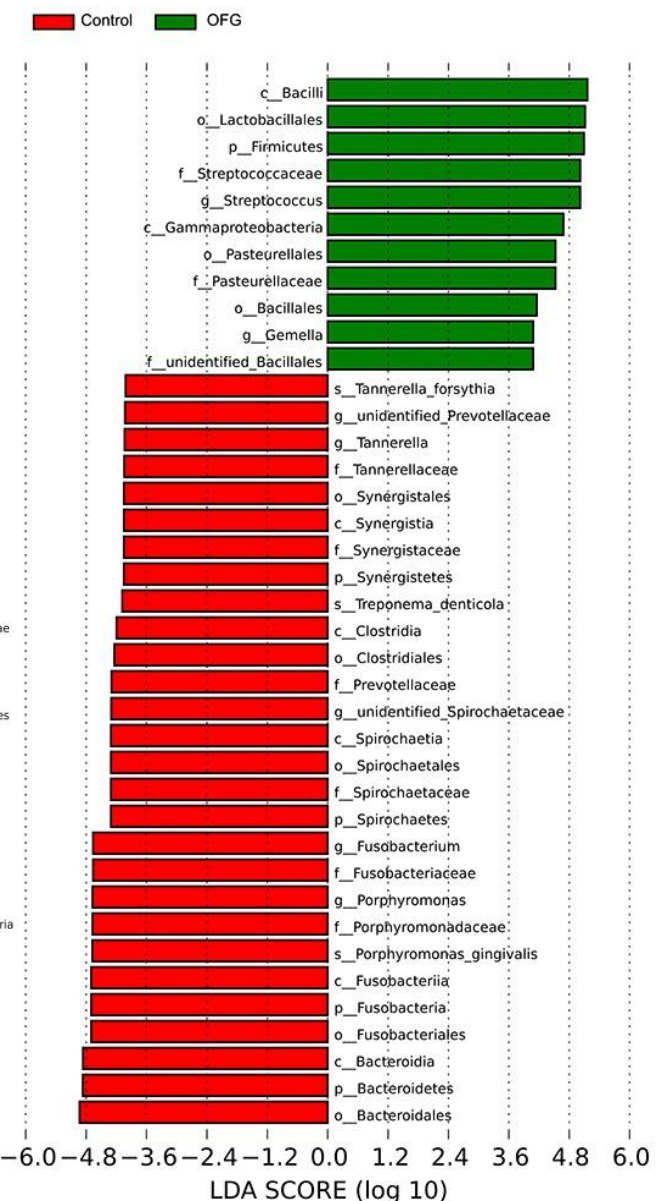

Figure 3. Differentially abundant taxa. (A) Phylums that were significantly more abundant between the AP bacterial communities of OFG patients and control individuals. (B) Taxa (highlighted by small circles and by shading) showing different abundance values in the two groups. (C) Taxa that were significantly more abundant between the two groups as identified by LDA effect size analysis: LDA score $\geq 4$. AP, apical periodontitis; OFG, orofacial granulomatosis; LDA, linear discriminant analysis . 


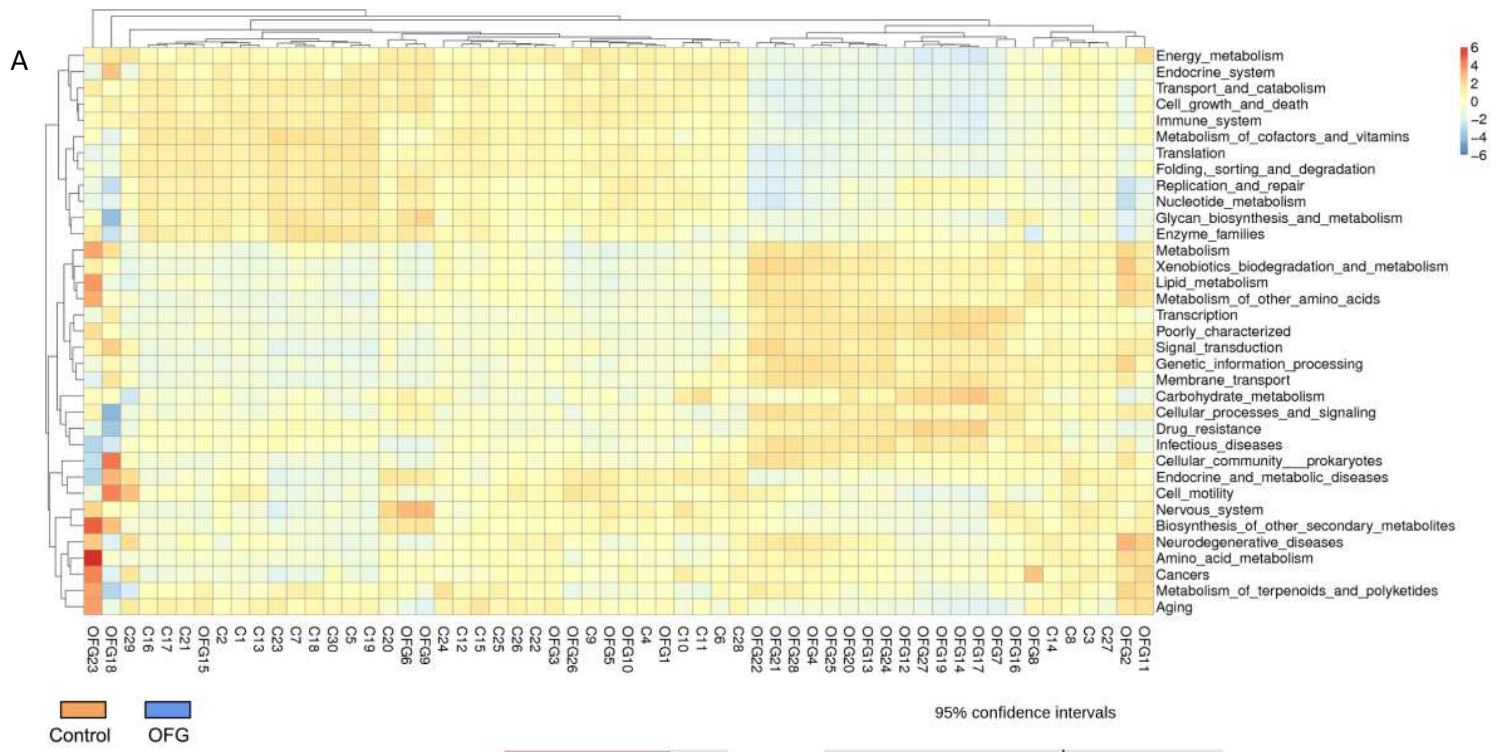

B
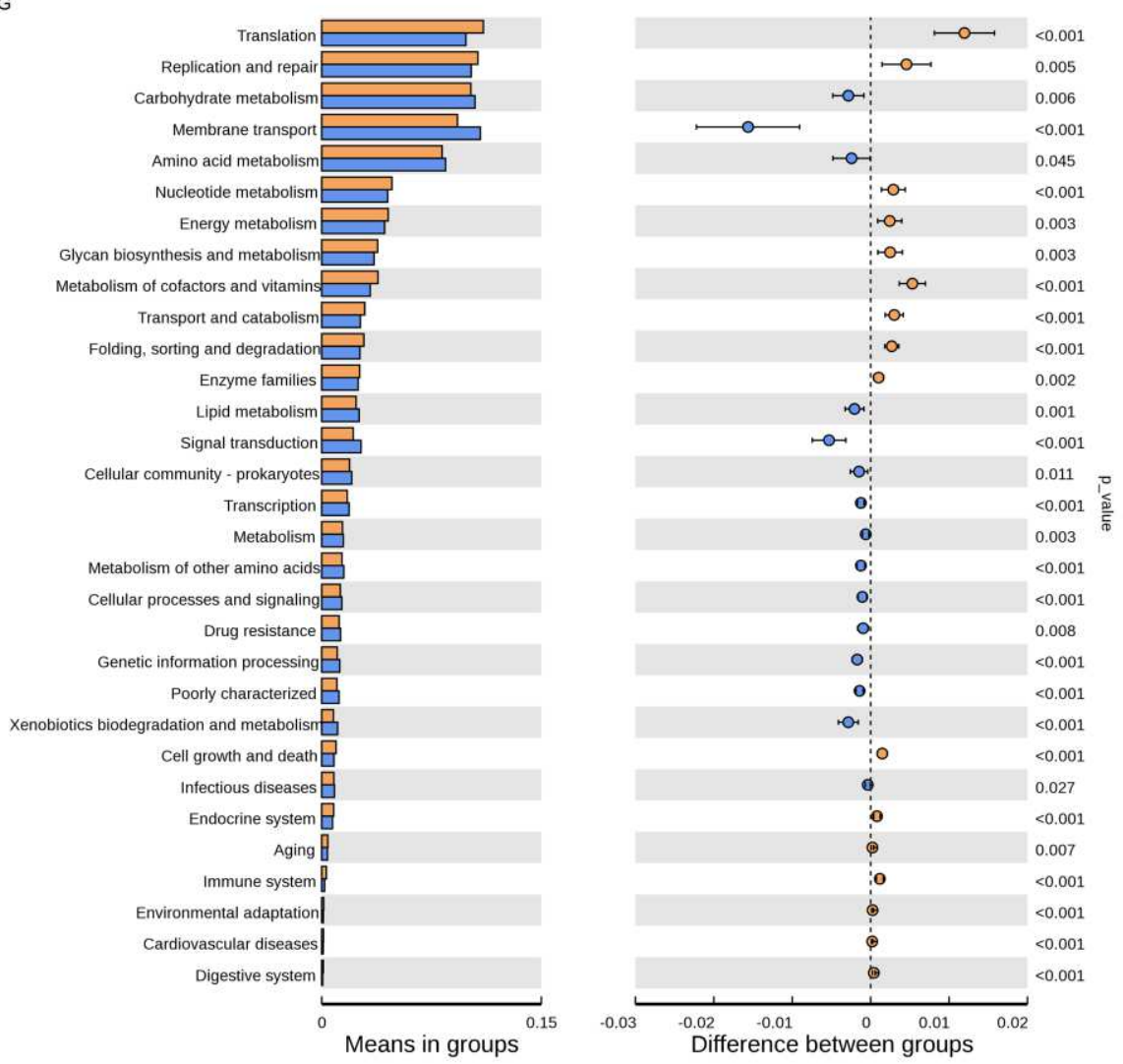

Figure 4. OFG patients and control individuals exhibited differential enrichment in AP microbial pathways. (A) Heat map for KEGG enrichment results. Metabolites are shown on the row in the format, sample IDs are shown on the column. (B) The significantly different microbial pathways between the two groups. Relative enrichment fold change is shown on the $x$ axis, and the name of pathway is shown on the $y$ axis. AP, apical periodontitis; OFG, orofacial granulomatosis. 

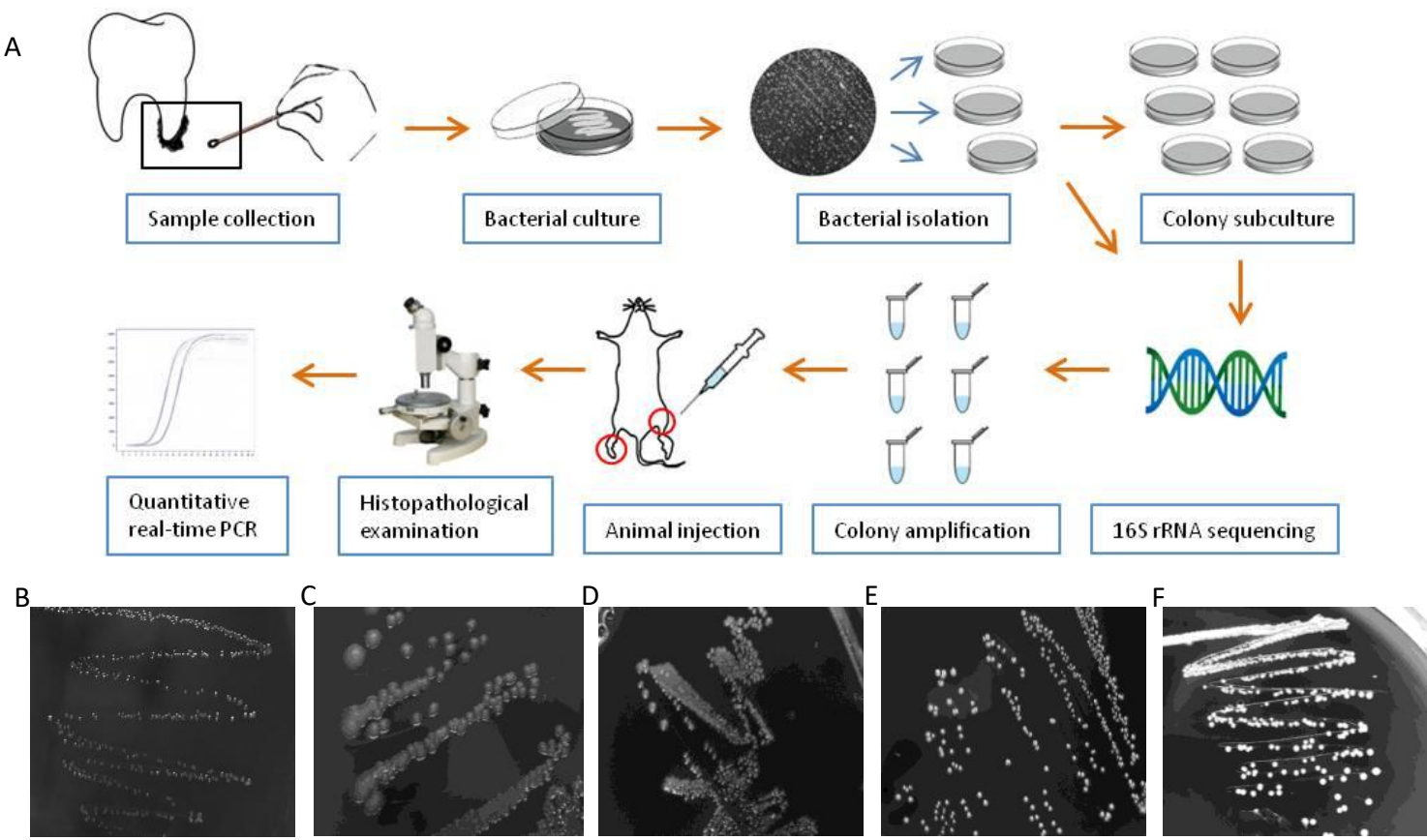

Figure 5. Bacterial isolation and animal experiments (A) The overall workflow in bacterial isolation, culture and animal experiment. The positive samples from animal were subjected to 16S rRNA sequence evaluation. (B) Growth of Streptococcus. (C) Growth of Neisseria subflava. (D) Growth of Actinomyces. (E) Growth of Lactobacillus casei. (F) Growth of Veillonella parvula. 


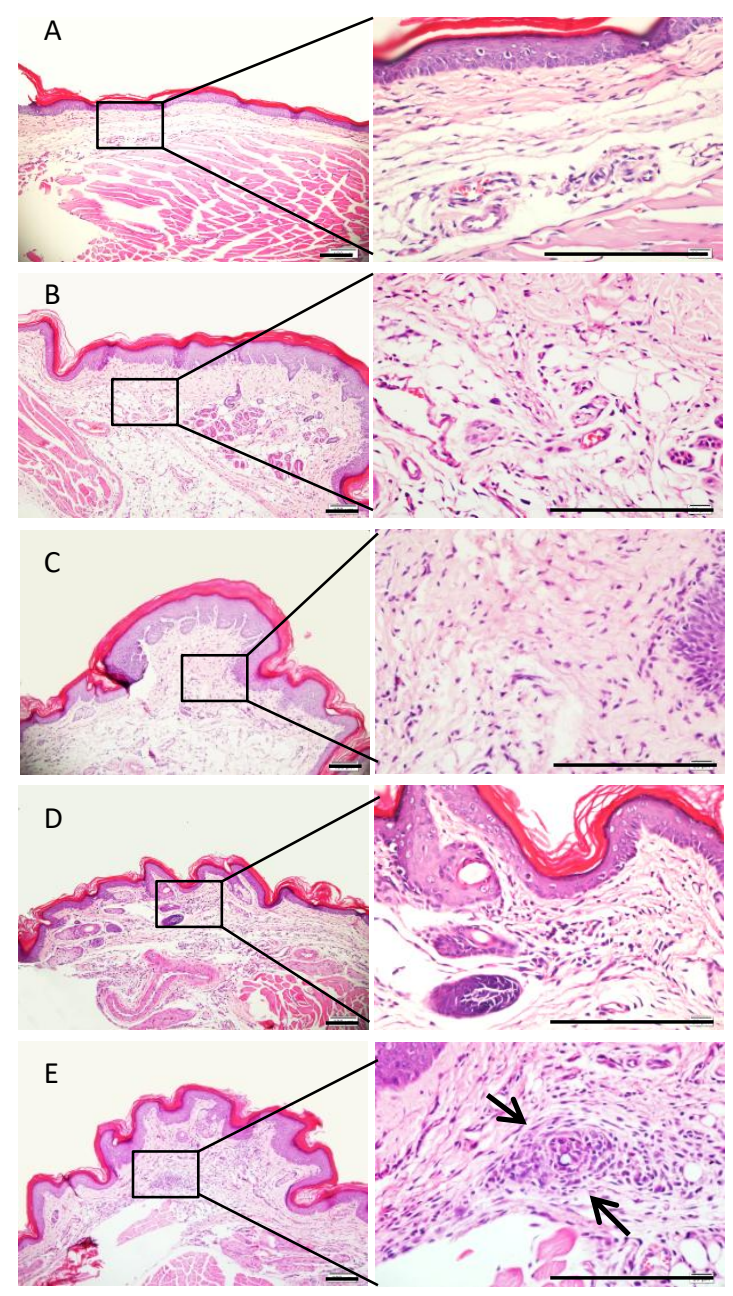

Figure 6. Photomicrograph of H\&E stained foot pad of the mice injected with different challenge bacteria. Histopathology of the foot pad injected with (A) Streptococcus, (B) Lactobacillus casei, (C) Veillonella parvula or (D) Actinomyces revealed relatively normal appearance of subcutaneous tissue. (E) Photomicrograph showed dermal inflammatory infiltration composed of epithelioid cells (arrows) in mice foot pads injected with Neisseria subflava.

Bar, $100 \mu \mathrm{m}$. 
Table 1: Distribution of AP bacteria at the phylum and genus (top 30) level in each group.

\begin{tabular}{|c|c|c|c|c|}
\hline \multirow{2}{*}{ Description } & \multicolumn{2}{|c|}{ Relative } & \multirow{2}{*}{ Level } & \multirow{2}{*}{$P$-value } \\
\hline & OFG & Control & & \\
\hline Actinobacteria & & & Phylum & \\
\hline Olsenella & $0.47 \%$ & $0.68 \%$ & genus & $\mathrm{N} / \mathrm{A}$ \\
\hline Actinomyces & $0.84 \%$ & $1.39 \%$ & genus & $\mathrm{N} / \mathrm{A}$ \\
\hline Corynebacterium & $0.27 \%$ & $0.50 \%$ & genus & $\mathrm{N} / \mathrm{A}$ \\
\hline Rothia & $0.37 \%$ & $0.31 \%$ & genus & $\mathrm{N} / \mathrm{A}$ \\
\hline Bacteroidetes & & & Phylum & \\
\hline Bacteroides & $0.24 \%$ & $0.53 \%$ & genus & 0.008 \\
\hline Odoribacter & $0.01 \%$ & $0.56 \%$ & genus & 0.029 \\
\hline Porphyromonas & $3.44 \%$ & $13.31 \%$ & genus & $<0.001$ \\
\hline Alloprevotella & $1.20 \%$ & $1.56 \%$ & genus & $\mathrm{N} / \mathrm{A}$ \\
\hline Prevotella & $3.69 \%$ & $5.13 \%$ & genus & $\mathrm{N} / \mathrm{A}$ \\
\hline unidentified_Prevotellaceae & $3.72 \%$ & $5.85 \%$ & genus & $\mathrm{N} / \mathrm{A}$ \\
\hline Tannerella & $0.22 \%$ & $2.40 \%$ & genus & $<0.001$ \\
\hline Phocaeicola & $0.13 \%$ & $0.72 \%$ & genus & 0.044 \\
\hline unidentified_Bacteroidales & $0.14 \%$ & $0.61 \%$ & genus & $\mathrm{N} / \mathrm{A}$ \\
\hline Capnocytophaga & $0.76 \%$ & $0.57 \%$ & genus & $\mathrm{N} / \mathrm{A}$ \\
\hline Salinimicrobium & $1.93 \%$ & $0.00 \%$ & genus & $\mathrm{N} / \mathrm{A}$ \\
\hline Firmicutes & & & Phylum & \\
\hline Gemella & $3.09 \%$ & $0.48 \%$ & genus & 0.01 \\
\hline Granulicatella & $1.56 \%$ & $0.04 \%$ & genus & 0.031 \\
\hline Lactobacillus & $3.75 \%$ & $0.39 \%$ & genus & $\mathrm{N} / \mathrm{A}$ \\
\hline Streptococcus & $25.09 \%$ & $2.74 \%$ & genus & $<0.001$ \\
\hline Oribacterium & $0.47 \%$ & $0.48 \%$ & genus & $\mathrm{N} / \mathrm{A}$ \\
\hline Filifactor & $0.62 \%$ & $1.31 \%$ & genus & 0.006 \\
\hline Parvimonas & $0.25 \%$ & $1.11 \%$ & genus & 0.024 \\
\hline unidentified_Clostridiales & $0.61 \%$ & $1.37 \%$ & genus & 0.002 \\
\hline Dialister & $1.04 \%$ & $1.25 \%$ & genus & $\mathrm{N} / \mathrm{A}$ \\
\hline Veillonella & $0.51 \%$ & $0.38 \%$ & genus & $\mathrm{N} / \mathrm{A}$ \\
\hline Fusobacteria & & & Phylum & \\
\hline Fusobacterium & $3.73 \%$ & $13.00 \%$ & genus & $<0.001$ \\
\hline Leptotrichia & $1.47 \%$ & $2.11 \%$ & genus & $\mathrm{N} / \mathrm{A}$ \\
\hline Proteobacteria & & & Phylum & \\
\hline Desulfomicrobium & $0.14 \%$ & $0.91 \%$ & genus & 0.012 \\
\hline Actinobacillus & $0.84 \%$ & $0.03 \%$ & genus & $\mathrm{N} / \mathrm{A}$ \\
\hline Aggregatibacter & $0.73 \%$ & $0.28 \%$ & genus & $\mathrm{N} / \mathrm{A}$ \\
\hline Lautropia & $0.52 \%$ & $0.36 \%$ & genus & $\mathrm{N} / \mathrm{A}$ \\
\hline Neisseria & $4.78 \%$ & $1.68 \%$ & genus & 0.049 \\
\hline Stenotrophomonas & $2.38 \%$ & $0.07 \%$ & genus & 0.038 \\
\hline Spirochaetes & & & Phylum & \\
\hline unidentified_Spirochaetaceae & $5.11 \%$ & $8.53 \%$ & genus & $\mathrm{N} / \mathrm{A}$ \\
\hline Synergistetes & & & Phylum & \\
\hline Fretibacterium & $0.34 \%$ & $2.26 \%$ & genus & $<0.001$ \\
\hline unidentified_Bacteria & & & Phylum & \\
\hline Campylobacter & $0.92 \%$ & $2.62 \%$ & genus & 0.007 \\
\hline Others & $13.16 \%$ & $7.31 \%$ & genus & $\mathrm{N} / \mathrm{A}$ \\
\hline
\end{tabular}




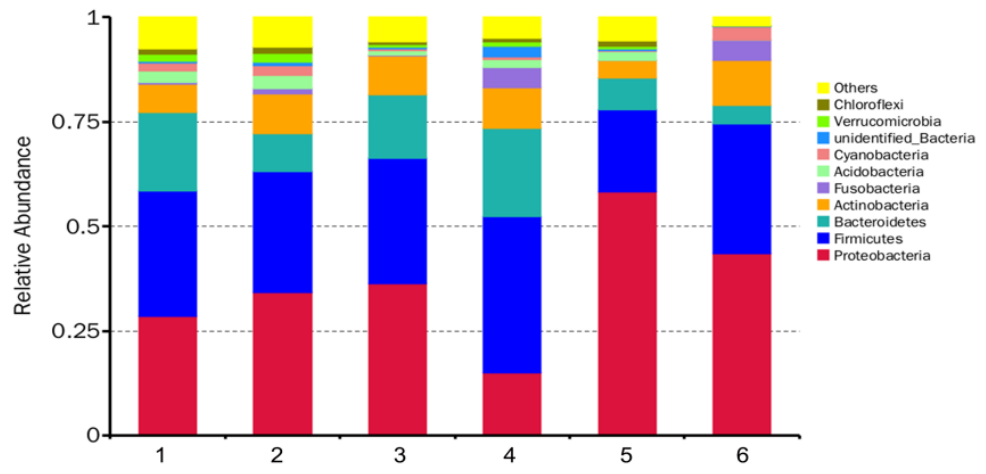

Figure Sup 1. Top 10 different bacterial phyla in the biopsy tissues of OFG patients.

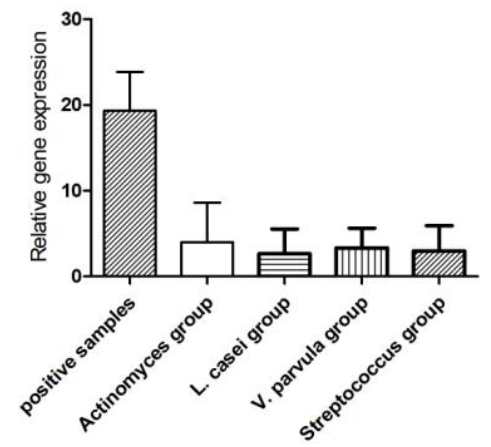

Figure Sup 2. N. subflava distribution among different samples analyzed by qPCR. 
Table S1: Distribution of bacteria at the genus level (top 10) in the lesion tissue of OFG patients.

\begin{tabular}{llllllll}
\hline Taxonomy & \multicolumn{2}{c}{ patient 1} & patient 2 & patient 3 & patient 4 & patient 5 & patient 6 \\
\hline Neisseria & $0.32 \%$ & $0.24 \%$ & $0.13 \%$ & $0.57 \%$ & $0.10 \%$ & $34.34 \%$ \\
Enhydrobacter & $0.57 \%$ & $0.79 \%$ & $13.88 \%$ & $0.18 \%$ & $0.22 \%$ & $0.02 \%$ \\
Streptococcus & $0.63 \%$ & $0.81 \%$ & $0.58 \%$ & $0.69 \%$ & $1.09 \%$ & $13.60 \%$ \\
Pseudomonas & $0.96 \%$ & $1.11 \%$ & $0.68 \%$ & $0.73 \%$ & $12.71 \%$ & $0.59 \%$ \\
Lactobacillus & $4.24 \%$ & $11.04 \%$ & $7.25 \%$ & $5.51 \%$ & $3.17 \%$ & $0.59 \%$ \\
Delftia & $3.56 \%$ & $8.16 \%$ & $1.02 \%$ & $0.33 \%$ & $0.59 \%$ & $0.03 \%$ \\
Geobacter & $0.10 \%$ & $0.07 \%$ & $0.24 \%$ & $0.11 \%$ & $7.95 \%$ & $0.00 \%$ \\
Bacteroides & $5.37 \%$ & $1.00 \%$ & $7.13 \%$ & $3.03 \%$ & $1.36 \%$ & $0.15 \%$ \\
Rothia & $0.10 \%$ & $0.03 \%$ & $0.25 \%$ & $0.03 \%$ & $0.06 \%$ & $4.54 \%$ \\
Actinomyces & $0.08 \%$ & $0.31 \%$ & $0.41 \%$ & $0.59 \%$ & $0.14 \%$ & $4.54 \%$ \\
Others & $84.07 \%$ & $76.44 \%$ & $68.43 \%$ & $88.22 \%$ & $72.62 \%$ & $41.60 \%$ \\
\hline
\end{tabular}


Table S2: Occurrence frequency of organism in relation to isolates

\begin{tabular}{|c|c|c|c|}
\hline Organism & $\begin{array}{l}\text { Frequency/ } \\
\text { Percentage (\%) }\end{array}$ & Organism & $\begin{array}{l}\text { Frequency/ } \\
\text { Percentage (\%) }\end{array}$ \\
\hline Streptococcus & $30 / 100$ & Bifidobacterium & $4 / 14.3$ \\
\hline Neisseria & $26 / 85.7$ & Collinsella & $4 / 14.3$ \\
\hline Actinomyces & $21 / 71.4$ & Dialister & $4 / 14.3$ \\
\hline Haemophilus & $21 / 71.4$ & Eikenella & $4 / 14.3$ \\
\hline Veillonella & $21 / 71.4$ & Prevotella & $4 / 14.3$ \\
\hline Romboutsia & $19 / 64.3$ & Agathobacter & $2 / 7.1$ \\
\hline Blautia & $17 / 57.1$ & Bacteroides & $2 / 7.1$ \\
\hline Escherichia & $17 / 57.1$ & Capnocytophaga & $2 / 7.1$ \\
\hline Faecalibacterium & $17 / 57.1$ & Gemella & $2 / 7.1$ \\
\hline Lactobacillus & $13 / 42.9$ & Granulicatella & $2 / 7.1$ \\
\hline Campylobacter & $9 / 28.6$ & Intestinimonas & $2 / 7.1$ \\
\hline Fusobacterium & $9 / 28.6$ & Klebsiella & $2 / 7.1$ \\
\hline unidentified_Lachnospiraceae & $9 / 28.6$ & Peptoclostridium & $2 / 7.1$ \\
\hline Enterococcus & $6 / 21.4$ & Porphyromonas & $2 / 7.1$ \\
\hline Pantoea & $6 / 21.4$ & Ruminococcus & $2 / 7.1$ \\
\hline Roseburia & $6 / 21.4$ & & \\
\hline
\end{tabular}

